\title{
Biomaterials Research and Medical Applications
}

\author{
Vizureanu Petrica* \\ Technical University Iasi, Romania \\ *Corresponding author: Vizureanu Petrica, Technical University Iasi, Romania. \\ To Cite This Article: Vizureanu Petrica. Biomaterials Research and Medical Applications. Am J Biomed Sci \& Res. 2019 - 1(6). AJBSR. \\ MS.ID.000555. DOI: 10.34297/AJBSR.2019.01.000555
}

Received: March 05, 2019 | Published: March 15, 2019

\section{Introduction}

The biocompatibility of a material can be defined in the sense that it produces desired or tolerated reactions in a living organism. The metals, in contact with the biological body, give complex effects, producing a series of biological reactions depending on the concentration, the exposure time, etc. According to the biological interaction mode, the metals are divided into: (i) Metallic elements needed in very small concentrations for the living body called essential elements, including cobalt, manganese, zinc, magnesium, sodium, potassium etc.; (ii) Elements that produce toxic effects for the body if present in higher concentrations, the cytotoxic effect being demonstrated by the cell culture system, such as arsenic, cobalt, nickel etc . and (iii) Metals with allergic potential (nickel, cobalt and chromium) are considered to be highly allergic to the body. The overall strategy for testing biomaterials involves assessing them in two ways: (i) "in vitro" evaluation performed on cell cultures or in the blood; (ii) "in vivo" assessment on animals.

The mechanical properties of biomaterials are dependent on the chemical composition, structure and function of the implant in the living organism, and have corresponding properties similar to those of the tissue they are replacing.

Corrosion of implants has the effect the release of metal ions in the body, considered responsible for producing allergies and toxic reactions in the human body. For most alloys used in implanted medical applications, the rate of corrosion is mainly dependent on the properties and stability of the passive film formed at its surface. The quality of passive film protection is dependent on its ability to resist chemical / electrochemical degradation caused by the human body's environment, as well as its ability to repack quickly enough to avoid initiating attack of the metal substrate.

The use of biomaterials in medical applications contain several areas: orthopedics, cardiovascular surgery, ophthalmology, dentistry, urology, aesthetic surgery, neurology, suture material for wound healing, controlled drug delivery systems.

\section{Conclusion}

As in conclusion, designing and selecting biomaterials consists in choosing the correct material compositional limits, to not occur unwanted reactions through human tissue implant interaction.

The design of a material for use in medical applications takes into account biocompatibility, mechanical properties and corrosion resistance. For its functionality,apart from the aspects mentioned, is addedand the surgical technique applied to implantation and, last but not least, the patient's state of health. 